\title{
Integrating microRNA and messenger RNA expression profiles in a rat model of deep vein thrombosis
}

\author{
QIAN-QIAN JIN $^{1 *}$, JUN-HONG SUN ${ }^{1 *}$, QIU-XIANG DU ${ }^{1}$, XIAO-JUN LU ${ }^{1}$, XI-YAN ZHU ${ }^{1}$, \\ HAO-LIANG FAN $^{1}$, CHRISTIAN HÖLSCHER ${ }^{2}$ and YING-YUAN WANG ${ }^{1}$ \\ ${ }^{1}$ Department of Forensic Pathology, Shanxi Medical University, Taiyuan, Shanxi 030001, P.R. China; \\ ${ }^{2}$ Biochemical and Life Sciences, Lancaster University, Lancaster, Lancashire LA1 4YQ, UK
}

Received May 13, 2017; Accepted August 21, 2017

DOI: $10.3892 /$ ijmm.2017.3105

\begin{abstract}
Deep vein thrombosis (DVT) is a disease involving multiple genes and systems. MicroRNAs (miRNAs) represent a class of non-coding small RNAs that post-transcriptionally suppress their target genes. The expression patterns of miRNA and messenger RNA (mRNA) in DVT remain poorly characterized. The aim of the present study was to evaluate miRNA and mRNA expression profiles in a stasis-induced DVT rat model. Male SD rats were randomly divided into three groups as follows: DVT, sham and control. The inferior vena cava (IVC) of rats was ligated to construct stasis-induced DVT models. Rats were sacrificed three days after ligation, and morphological changes in the vein tissues were observed by hematoxylin and eosin and Masson staining. The miRNA and mRNA expression profiles were evaluated by microarrays, followed by bioinformatics analysis. The microarray analysis identified 22 miRNAs and 487 mRNAs that were significantly differentially expressed between the experimental and control groups, and between the experimental and sham groups, but not between the control and sham groups ( $\mathrm{P} \leq 0.05 ; \geq 2.0$-fold change). By subsequent bioinformatics analysis, a 19 miRNA-98 mRNAs network was constructed in the stasis-induced DVT rat model. Notably, the majority of these miRNAs and mRNAs are reported to be expressed by endothelial cells (ECs) and are associated with the function of ECs. The results provide evidence indicating that the regulatory association of miRNA and mRNA points to key roles played by ECs in thrombosis. These findings advance our understanding of the molecular regulatory mechanisms underlying the pathophysiology of DVT.
\end{abstract}

Correspondence to: Mr. Jun-Hong Sun or Dr Ying-Yuan Wang, Department of Forensic Pathology, Shanxi Medical University, 56 South Xinjian Road, Taiyuan, Shanxi 030001, P.R. China

E-mail: junhong.sun@sxmu.edu.cn

E-mail:wyy580208@163.com

*Contributed equally

Key words: deep vein thrombosis, bioinformatics, microarray, microRNA, messenger RNA

\section{Introduction}

Deep vein thrombosis (DVT) refers to the formation of a blood clot within a deep vein, predominantly in the legs. DVT and pulmonary embolism (PE) constitute a single disease process, termed venous thromboembolism (VTE), which is the third most common vascular disease in the United States (1). As the disease has an insidious onset and there is no obvious clinical symptom or sign in the early stages, the rate of misdiagnosis is high. When timely diagnosis and effective therapy are missed, DVT may lead to the abnormal swelling and ulceration of lower limbs, post-thrombotic syndrome and PE $(1,2)$. The incidence of VTE is $>1,000$ per year in the United States (3). Thus, DVT is a major cause of mortality and leads to significant morbidity. In forensic practice, $\mathrm{PE}$ is a major cause of sudden death and is attributable primarily to DVT (4). Studies of DVT improve the understanding of the disease, as well as increase the successful rescue rate and detection rate, which is significant in clinical diagnosis treatment and forensic identification.

In recent years, with the deepening of theoretical research into thrombosis, DVT has been recognized as a disease that involves multiple factors and systems (5). Due to the complexity of the disease, traditional Northern blotting and quantitative polymerase chain reaction methods have been unable to fully elucidate its mechanisms. To develop a more comprehensive understanding of DVT, high-throughput microRNA (miRNA) and messenger RNA (mRNA) microarray technology were used in the present study.

miRNAs represent a class of non-coding small RNAs that post-transcriptionally suppress their target genes (6). There is increasing evidence that miRNA expression patterns change in many vascular diseases (7-10). Although DVT has been investigated extensively, the molecular mechanisms underlying the pathophysiological changes remain to be defined. Furthermore, information on changes in miRNA expression within the vessel tissues is limited and, to date, to the best of our knowledge, there are no studies describing miRNA-mRNA interactions in DVT.

In the present study, miRNA and mRNA expression in vessel tissue samples from rat DVT models were assessed by microarray. Furthermore, bioinformatics analyses were used to build and analyze the miRNA-mRNA network. The present findings provide systematic and comprehensive insights 
into the molecular mechanisms of DVT. The study design is presented in Fig. 1.

\section{Materials and methods}

Animal model of venous thrombosis. All experiments were reviewed and approved by the ethics committee of the Institute of Laboratory Animal Science of Shanxi Medical University (Taiyuan, China).

Adult male Sprague Dawley (SD) rats $(n=36$; Shanxi Laboratory Animal Center, Taiyuan, China) 8-10 weeks of age and weighing 280-300 g were used in the present study. The rats were divided into three groups as follows: DVT, sham and control $(n=12)$. The rats were anesthetized by $10 \%$ chloral hydrate. A midline laparotomy was performed. The inferior vena was explored by moving the small intestine out of the way, and all side branches were ligated. IVC was ligated just below the left renal vein. A microvascular clamp was attached to the confluence of iliac veins for $15 \mathrm{~min}$. The skin was sutured by 3-0 Prolene suture and penicillin powder (Sigma, Washington, DC, USA) covering the incision evenly was used. The sham-surgery rats received anesthesia and all surgical procedures, but without IVC ligation or clamping. The control group received no treatment.

Tissue harvesting. The rats were sacrificed at day 3 after ligation. The IVC with thrombus was carefully harvested. One part of the tissue was fixed in $10 \%$ formalin solution for histological analysis and the rest was stored in RNAsafety (Shanghai Biotechnology Corp., Shanghai, China) for microarray analyses.

Histological analysis. For histological examinations, the IVC tissue was fixed with $4 \%$ paraformaldehyde for $48 \mathrm{~h}$ and embedded in paraffin wax. The tissues were then cut into $4-\mu \mathrm{m}$-thick sections and the sections were dewaxed in xylene twice at $37^{\circ} \mathrm{C}$ for $15 \mathrm{~min}$ each time, rehydrated through decreasing concentrations of ethanol, and washed in distilled water at room temperature for $5 \mathrm{~min}$ each time. They were finally stained with hematoxylin and eosin (H\&E) or Masson's trichrome, both following the company's instructions (Nanjing Jiancheng Bioengineering Institute, Nanjing, China). For H\&E staining, the slides were dipped into the jar containing hematoxylin for $5 \mathrm{~min}$ and with eosin for $30 \mathrm{sec}$ with agitation at room temperature. For Masson staining, slides were stained at room temperature with nuclear staining solution for $1 \mathrm{~min}$, with cytoplasmic stain solution for $45 \mathrm{sec}$, then washed with phosphomolybdic acid for $6 \mathrm{~min}$ and last counterstained for $5 \mathrm{~min}$. After staining, specimens were observed under a light microscope (Panoramic SCAN II; 3DHISTECH Kft., Budapest, Hungary) to evaluate the histomorphology of the venous walls.

RNA isolation and quantification. Total RNA was extracted and purified using an Ambion mirVana miRNA isolation kit (cat. no. AM1561; Thermo Fisher Scientific, Inc., Waltham, MA, USA) and RNA integrity was assessed using an Agilent Bioanalyzer 2100 (Agilent Technologies, Inc., Santa Clara, CA, USA). The RNA samples were used for microarrays. To avoid differences between individuals, $200 \mathrm{ng}$ total RNA isolated from three rats per group was pooled into a single sample. Microarray experiments were repeated to produce three independent biological replicates.

miRNA microarray experimental set-up and initial data analysis. miRNA expression analysis was performed using nine Agilent rat miRNA $(8 \times 60 \mathrm{~K})$ V21.0 microarrays (design ID: 70154; Agilent Technologies, Inc.) at the National Engineering Center for Biochip, Shanghai Biotechnology, Corp. (Shanghai, China) miRNA was labeled using the miRNA complete labeling and hybridization kit (cat. no. p/n 5190-0456; Agilent Technologies, Inc.). The kit was used to hybridize each slide with Cy3-labeled RNA in a hybridization oven. Subsequent to hybridization, slides were washed and scanned using a Microarray Scanner (cat. no. G2565CA; Agilent Technologies, Inc.) using Feature Extraction software (version 10.7; Agilent Technologies, Inc.).

Raw data were normalized using the quantile algorithm in the GeneSpring software (version 12.6; Agilent Technologies, Inc.). The results of signal values are presented as means \pm standard deviation. Student's t-test was used to identify differences between groups using the $\mathrm{R}$ package. Furthermore, the fold change was the ratio of the mean values of two comparative groups. miRNAs with fold differences $\geq 2.0$ and $\mathrm{P} \leq 0.05$ were considered to indicate a statistically significant difference.

mRNA microarray experimental set-up and initial data analysis. mRNA expression analysis was performed using nine Agilent Whole Rat Genome Microarrays 4x44K (design ID:014879; Agilent Technologies, Inc.) at the National Engineering Center for Biochip, Shanghai Biotechnology Corp. Total RNA was amplified and labeled using the Low Input Quick Amp Labeling kit, One-Color (cat. no. 5190-2305; Agilent Technologies, Inc.). Labeled cRNA was purified using an RNeasy mini kit (cat. no. 74106; Qiagen GmbH, Hilden, Germany). The left process was similar to miRNA microarray. mRNAs with fold differences $\geq 2.0$ and $\mathrm{P} \leq 0.05$ were considered to indicate a statistically significant difference.

Integrated analysis of miRNA and mRNA expression profiles. Five prediction tools: TargetMiner (http://www.isical. ac.in/ bioinfo_miu/targetminer20.htm), miRDB (http://mirdb. org/miRDB/index.html), microRNA (http://www.microrna. org/microrna/home.do), TarBase (http://diana.imis.athenainnovation.gr/DianaTools/index.php?r=tarbase/index), and RNA22 (https://cm.jefferson.edu/rna22/) quoted by miRBase database, were used to obtain the target genes of differentially expressed miRNAs. All of the tools were assembled into an online tool (http://www.shbio.com/analysis.html).

The predicted mRNA targets were then compared with experimentally determined mRNAs by microarray. Subsequently, according to Pearson's correlation coefficients and the associations of mRNAs in STRING (http://string-db.org/), a regulatory network was determined, which was comprised of 19 miRNAs and 98 mRNAs.

These 98 mRNAs were uploaded to Database for Annotation, Visualization and Integrated Discovery (version 6.7; https://david. ncifcrf.gov/) for Gene Ontology (GO) functional annotation. The Kyoto Encyclopedia of Genes and Genomes (KEGG) pathway database (http://www.genome.jp/kegg/kegg1.html) was used to identify the enriched pathways of targets. Additionally, 
Three groups: DVT, Sham, Control

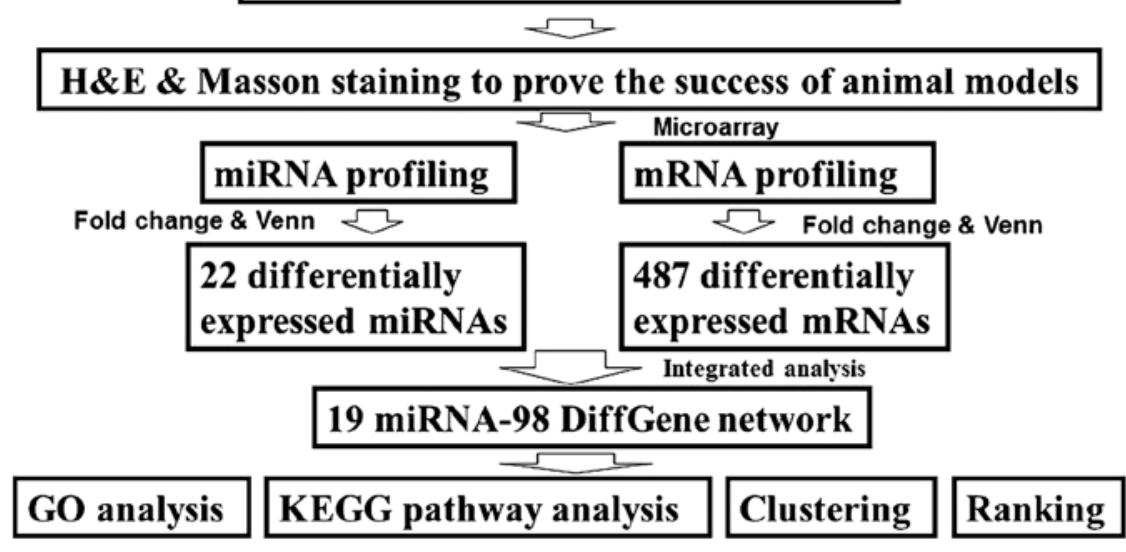

Figure 1. Flow chart of the experimental design.
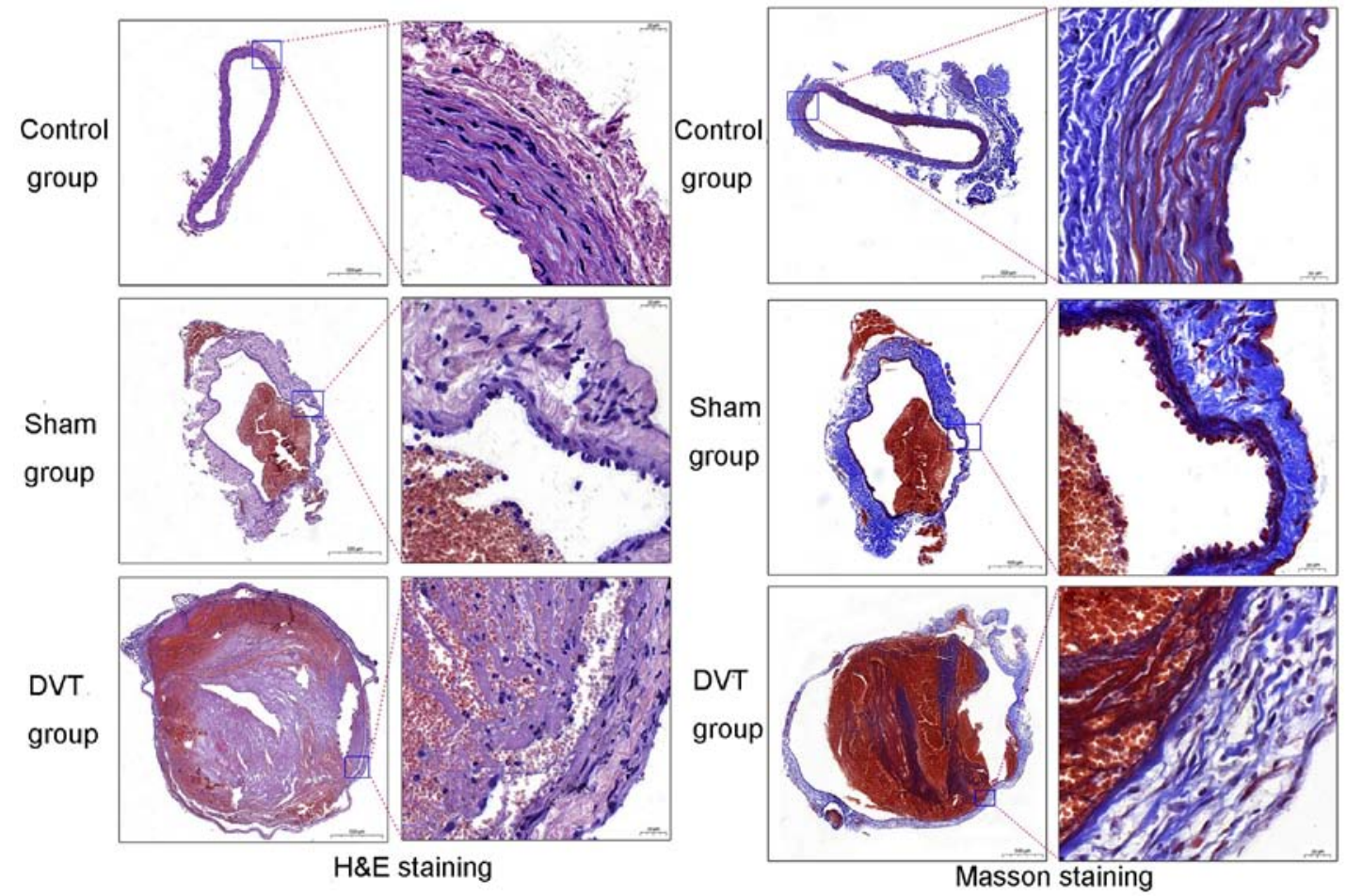

Figure 2. Photomicrographs of the sections stained with H\&E and Masson's trichrome. H\&E and Masson staining exhibiting normal inferior vena cava in the control group. Accumulation of blood cells is apparent in the veins of the sham group, while no fibrin is observed. No thrombosis is present in the control and sham groups. Vascular endothelial cells in the DVT group are not continuous, and the images exhibit substantial numbers of inflammatory cells infiltrating and surrounding the blood vessels. The thrombus in the lumen is a mixed thrombus. Collagen fiber hyperplasia occurred and partially entered the thrombus. H\&E, hematoxylin and eosin; DVT, deep vein thrombosis. Scale bars of the left pictures of H\&E or Masson staining represents $500 \mu \mathrm{m}$; scale bars of the right pictures represents $20 \mu \mathrm{m}$.

19 miRNAs were subjected to a two-way unsupervised hierarchical clustering analysis using MultiExperiment Viewer software 4.7.4, a part of the TM4 Microarray software suite (Dana-Farber Cancer Institute, Boston, MA, USA) and ranked according to fold change and degrees in the network.

Statistical analysis. Data are presented as the means \pm SD. Statistical difference $(\mathrm{P} \leq 0.05)$ was calculated by Student's t-test and correlations were evaluated by Pearson's correlation coefficients using the $\mathrm{R}$ package. The $\mathrm{P}$-value $<0.05$ was considered statistically significant.

\section{Results}

Histological changes in veins following ligation. Sections of the IVC were stained with H\&E and Masson's trichrome and observed under a light microscope. Clear differences were observed among the three groups (Fig. 2). With discontinuous vascular endothelial cells (ECs), numerous inflammatory cells infiltrated and entered the blood vessels, demonstrating a stable mixed thrombus following treatment in the DVT group. The staining results indicated the success of the SD DVT models. 

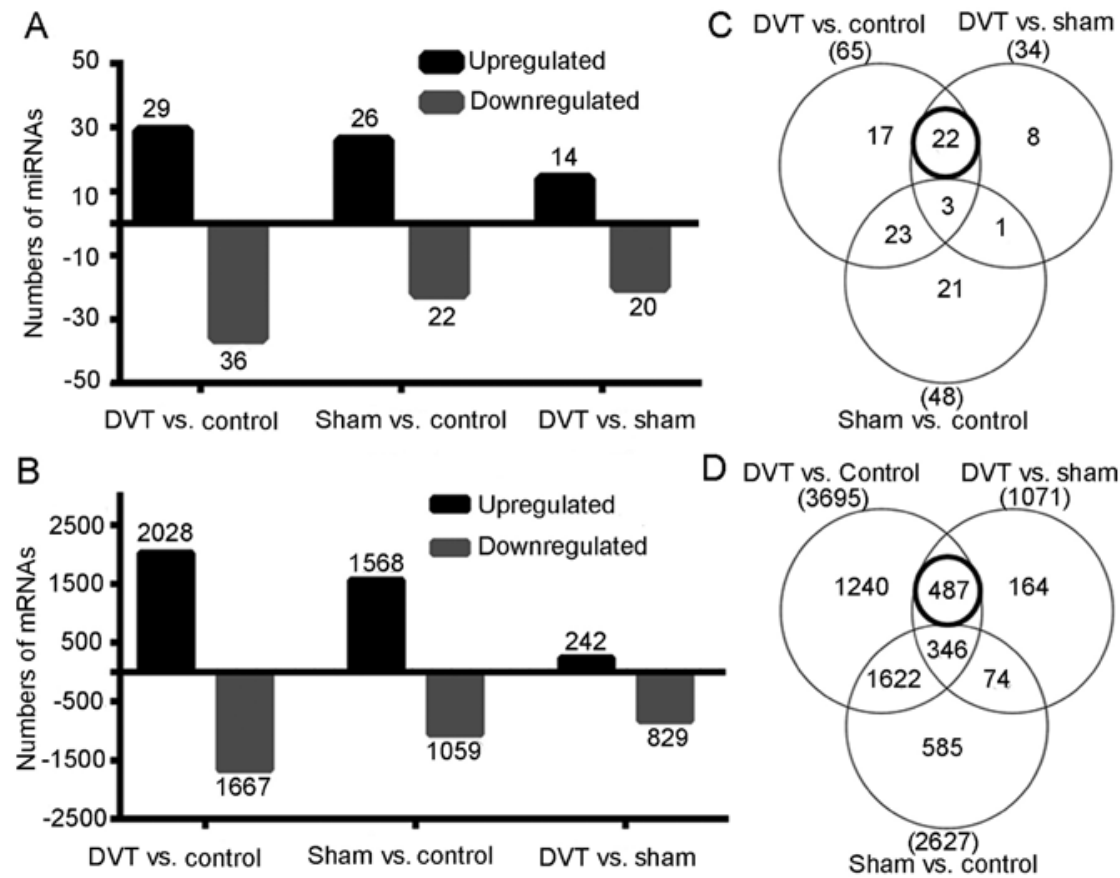

Figure 3. Analysis of mRNA and miRNA expression in rat veins. Bar charts indicating the numbers of upregulated and downregulated (A) miRNAs and (B) mRNAs among the DVT, sham, and control groups. (C and D) Venn diagrams of the numbers of differentially expressed genes from comparisons of the DVT, sham, and control groups. mRNAs and miRNAs with 2-fold differences and statistical significance $(\mathrm{P}<0.05)$ were identified as differentially expressed mRNAs or miRNAs. The numbers in the overlapping areas (bold circles) represent differentially expressed miRNAs and mRNAs analyzed in the subsequent step. Differences were observed between the DVT group and the two other groups, but not between the control and sham groups. mRNA, messenger RNA; miRNA, microRNA.

miRNA expression profiling. To evaluate the role of miRNAs in thrombosis, the miRNA profiling in the DVT animal models were investigated, along with the sham-surgery and control rats $(\mathrm{n}=9)$ by microarray. Among 758 detectable miRNAs, 65 miRNAs (29 upregulated and 36 downregulated) significantly difference between the DVT and control groups, 34 miRNAs (14 upregulated and 20 downregulated) between the DVT and sham groups, and 48 miRNAs (26 upregulated and 22 downregulated) between the sham and control groups using a cut-off with an adjusted $\mathrm{P} \leq 0.05$ and a fold-change of $\geq 2.0$ (Fig. 3A). To exclude any effects of the surgical procedure, only 22 differentially expressed miRNAs in the overlapping areas (Fig. 3C) were focused upon, indicating differences between the DVT and the two other groups, but no difference between the control and sham groups. These 22 miRNAs were subjected to further analyses.

$m R N A$ expression profiling. More than 41,000 rat genes and transcripts were investigated in the present study. Of them, 3,695 genes (2,028 upregulated and 1,667 downregulated) differed significantly between the DVT and control groups, 2,627 genes (1,568 upregulated and 1,059 downregulated) between the sham and control groups, and 1,071 genes (242 upregulated and 829 downregulated) between the DVT and sham groups using a cut off with an adjusted $\mathrm{P} \leq 0.05$ and a fold-change of $\geq 2.0$ (Fig. 3B). Similarly, 487 differentially expressed genes (DEGs) in overlapping areas (Fig. 3D) were selected for further analyses.

miRNA-mRNA network. The 22 miRNAs were integrated to the experimentally determined 487 DEGs to obtain the
miRNA-mRNA network (Fig. 4), which was composed of 19 miRNAs (6 upregulated and 13 downregulated) and 98 differentially expressed mRNAs (13 upregulated and 85 downregulated).

Functional classification of 98 genes. GO assigns biological processes (BP), cellular components (CC) and molecular function (MF). The BP analysis involved 83 GO terms; five of the top 10 were associated with adenylate cyclase activity, and two were associated with cell growth (Fig. 5A). Seven were found in $\mathrm{CC}$, indicating that the DEGs were predominantly extracellular genes (45.66\%) and plasma membrane genes (34.78\%) (Fig. 5B). In seven MF annotations, the majority of genes belonged to binding activity genes (18.4\%), while active protein genes represented $8.7 \%$ (Fig. 5C).

To evaluate the roles of the target genes in DVT, six specific terms (angiogenesis, cell proliferation, adhesion, inflammatory response, apoptosis and hypoxia) mediating vascular function were extracted from multiple levels within the GO hierarchy (Table I). Notably, the majority of these genes were closely associated with endothelial function (marked in bold in Table I) and the majority demonstrated a decreased expression level in DVT. For example, the expression levels of EC proliferative and anti-apoptosis genes, growth arrest specific 6 (GAS6), vascular endothelial growth factor B (VEGFB), the most well-known inducer of angiogenesis, and endothelial inflammation factor, Kruppel like factor 4 (KLF4) were reported to be reduced in atherosclerosis (11). In addition, adhesion-associated genes, including junctional adhesion molecule 2 (JAM2), which affects EC junctions (12), C-X-C motif chemokine ligand 12 (CXCL12), which mediates 


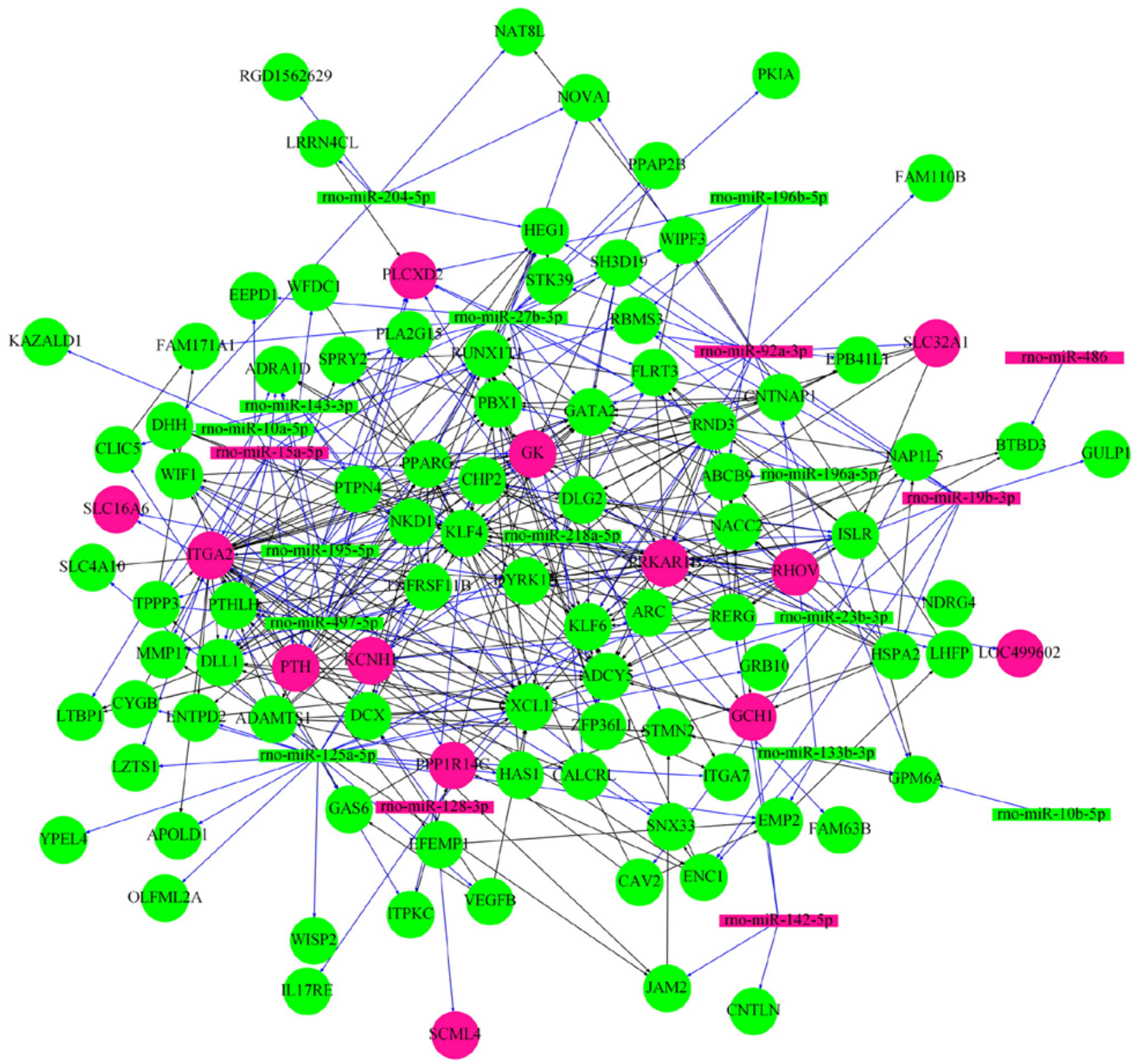

Figure 4. miRNAs-mRNAs network in deep vein thrombosis. The 19 miRNAs-98 mRNAs network was obtained by integrating the miRNA and mRNAs profiling. Pink rectangle, 6 upregulated miRNAs; green rectangle, 13 downregulated miRNAs; pink circle, 13 upregulated mRNAs; green circle, 85 downregulated mRNAs; blue line, regulatory association of miRNA and mRNA by Pearson's correlation coefficients; black line, regulatory association of mRNA and mRNA in STRING. miRNA, microRNA; mRNA, messenger RNA.

recruitment of inflammatory and thrombotic cells (13), and apolipoprotein L domain containing 1 (APOLD1) (14), a novel EC early response protein associated with hypoxia, were equally reduced in DVT.

KEGG pathway analysis demonstrated that four of the top 15 signal pathways were associated with cardiovascular disease, and three to metabolism. Among them, the following three had enriched gene numbers $>3$ : dilated cardiomyopathy, vascular smooth muscle contraction and focal adhesion pathways (Fig. 5D).

Clustering of 19 miRNA expression. The clustering analysis of the nine samples and 19 differentially expressed miRNAs revealed a distinct miRNA signature during DVT (Fig. 6A). The six upregulated miRNAs grouped together and the 13 downregulated miRNAs were divided into two categories, in which miR-133b-3p, miR-218a-5p and miR-204-5p were grouped together, while the remaining 10 miRNAs all gather in a second cluster.

Ranking. To demonstrate the value of the 19 miRNAs in DVT, they were positioned according to the degree of co-expression from network (Table II) and fold change form profiling (Tables III and IV). The co-expression was ranked according to the degree of co-expression, the highest degree scored 19 points, and the lowest 1 point. The fold change score was ranked in the same way. The most differentially expressed upregulated miRNA received 6 points (for a total of 6 upregulated miRNAs) and downregulated miRNAs received 13 points (for a total of 13 downregulated miRNAs), and the lowest 1 point (data not shown). Bar charts of the rankings are presented in Fig. 6B and C. The top upregulated miRNA was miR-92a-3p, while miR-218a-5p was ranked first among the downregulated miRNAs. 
Table I. Genes associated with specific GO terms (angiogenesis, cell proliferation, adhesion, inflammatory response, apoptosis and hypoxia) in DVT. Genes related to endothelial cells are marked in bold.

\begin{tabular}{lll}
\hline $\begin{array}{l}\text { Specific Go terms } \\
\text { Angiogenesis }\end{array}$ & \multicolumn{1}{c}{ Upregulated genes } & \multicolumn{1}{c}{ Downregulated genes } \\
Cell proliferation & PTH, KCNH1, ITGA2 & $\begin{array}{l}\text { APOLD1, CALCRL, KLF4, DLL1, CXCL12, EMP2, GATA2, VEGFB } \\
\text { GAS6, GATA2, KLF4, NACC2, NDRG4, PBX1, PPARG, PTHLH, }\end{array}$ \\
& & RERG, SPRY2, TNFRSF11B, VEGFB, WFDC1, WISP2, ZFP36L1 \\
Adhesion & ITGA2 & PPAP2B, HSPA2, GAS6, ZFP36L1, CXCL12, JAM2, KLF4, EMP2, \\
& & FLRT3, WISP2, DLL1, CAV2, HAS1, ITGA, RND3, CNTNAP1 \\
Inflammatory response & ITGA2 & PPARG, CALCRL, WFDC1, STK39, TNFRSF11B, IL17RE \\
Apoptosis & PTH & ARC, HSPA2, GAS6, TNFRSF11B, NACC2, PPARG, SPRY2, \\
& & CXCL12, VEGFB, GULP1, KLF4, ZFP36L1, DLL1, STK39 \\
Hypoxia & APOLD1, CYGB, ARC, CXCL12
\end{tabular}

GO, Gene Ontology.

Table II. Degrees of differential expression and targets of the 19 differentially expressed miRNAs.

\begin{tabular}{|c|c|c|}
\hline miRNA & Degree & Targets \\
\hline $\operatorname{miR}-125 a-5 p$ & 22 & $\begin{array}{l}\text { ENTPD2, CXCL12, GAS6, YPEL4, ARC, CHP2, GRB10, ITGA7, CYGB, SLC4A10, HAS1, WISP2, } \\
\text { OLFML2A, LZTS1, TNFRSF11B, ADAMTS1, EMP2, APOLD1, RHOV, VEGFB, CLIC5, ITPKC }\end{array}$ \\
\hline $\operatorname{miR}-195-5 p$ & 20 & $\begin{array}{l}\text { NKD1, DLL1, PLCXD2, ADRA1D, GK, SLC16A6, WIF1, ITGA2, SNX33, PLA2G15, } \\
\text { FLRT3, TPPP3, MMP17, ADCY5, RUNX1T1, ISLR, DYRK1B, PTHLH, PTPN4, PTH }\end{array}$ \\
\hline $\operatorname{miR}-497-5 p$ & 19 & $\begin{array}{l}\text { DLL1, ITGA2, NKD1, GK, PTH, ADRA1D, ISLR, PLA2G15, PTHLH, RUNX1T1, SNX33, } \\
\text { TPPP3, MMP17, SLC16A6, PLCXD2, ADCY5, DYRK1B, WIF1, PTPN4 }\end{array}$ \\
\hline $\operatorname{miR}-27 b-3 p$ & 18 & $\begin{array}{l}\text { PPARG, RND3, ZFP36L1,PPAP2B, FAM171A1, EPB41L1, HEG1, FLRT3, GATA2, } \\
\text { NOVA1, STK39, EEPD1, WIPF3, SPRY2, PLCXD2, STMN2, DCX, PKIA }\end{array}$ \\
\hline $\operatorname{miR}-15 a-5 p$ & 12 & ISLR, RUNX1T1, DLL1, PTH, PTHLH, TPPP3, GK, NKD1, ITGA2, ADRA1D, PLA2G15, MMP17 \\
\hline $\operatorname{miR}-10 a-5 p$ & 9 & ADRA1D, RBMS3, LZTS1, KAZALD1, SH3D19, KLF4, EEPD1, LTBP1, PPP1R14C \\
\hline $\operatorname{miR}-218 a-5 p$ & 9 & FLRT3, SH3D19, PLA2G15, CALCRL, KCNH1, PLCXD2, NDRG4, FAM63B, LHFP \\
\hline $\operatorname{miR}-92 a-3 p$ & 9 & FAM110B, GATA2, NOVA1, SLC32A1, STK39, KLF4, PRKAR1B, KLF6, GPM6A \\
\hline $\operatorname{miR}-128-3 p$ & 8 & EFEMP1, DCX, SCML4, RND3, IL17RE, ITPKC, KLF4, ABCB9 \\
\hline $\operatorname{miR}-19 b-3 p$ & 8 & GULP1, SH3D19, HSPA2, RBMS3, HEG1, GRB10, ENC1, ARC \\
\hline $\operatorname{miR}-23 b-3 p$ & 7 & KCNH1, DLG2, CXCL12, LOC499602, CAV2, NACC2, NAP1L5 \\
\hline $\operatorname{miR}-204-5 p$ & 6 & NAT8L, LRRN4CL, DHH, NOVA1, HEG1, RGD1562629 \\
\hline $\operatorname{miR}-143-3 p$ & 5 & DYRK1B, CXCL12, CLIC5, WFDC1, PTPN4 \\
\hline $\operatorname{miR}-133 b-3 p$ & 4 & EMP2, ENC1, CNTNAP1, GPM6A \\
\hline $\operatorname{miR}-142-5 p$ & 4 & RERG, JAM2, CNTLN, GCH1 \\
\hline $\operatorname{miR}-196 a-5 p$ & 4 & PLCXD2, RBMS3, PBX1, ABCB9 \\
\hline $\operatorname{miR}-196 b-5 p$ & 4 & RBMS3, PLCXD2, PBX1, ABCB9 \\
\hline $\operatorname{miR}-10 b-5 p$ & 1 & GPM6A \\
\hline miR-486 & 1 & BTBD3 \\
\hline
\end{tabular}
junctional adhesion molecule 2; CXCL12, C-X-C motif chemokine ligand 12; APOLD1, apolipoprotein L domain containing 1; HSPA2, heat shock protein family A (Hsp70) member 2; DLL1, delta like canonical Notch ligand 1; GATA2, GATA binding protein 2; KLF4, Kruppel like factor 4; ITGA2, integrin subunit $\alpha 2$. 
A

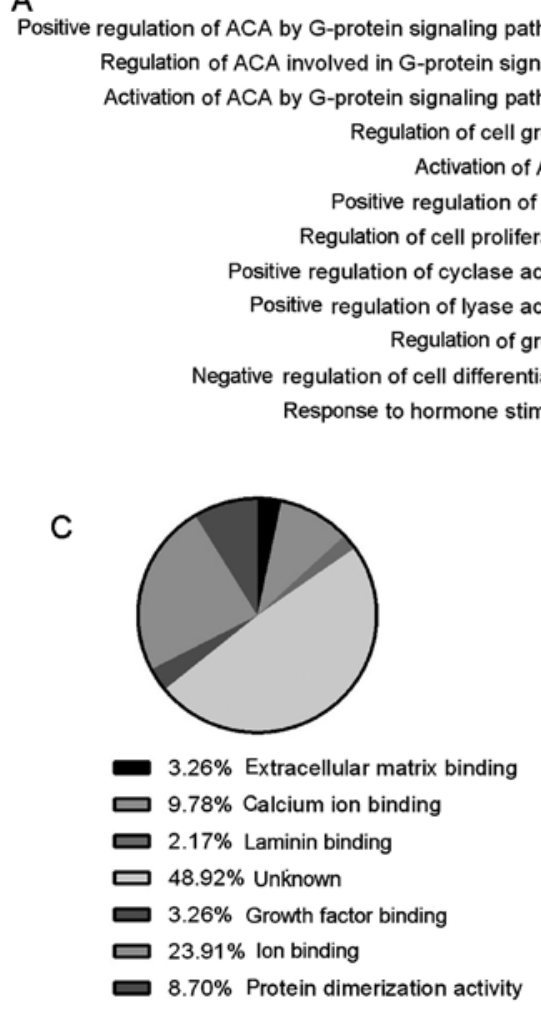

$\mathrm{B}$

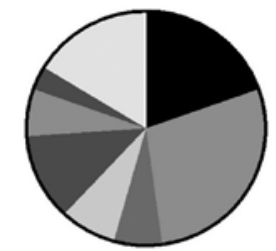

$19.57 \%$ Extracellular region

๑ $28.26 \%$ Plasma membrane

$6.52 \%$ Internal side of plasma membrane

$7.61 \%$ Extracellular matrix

$11.96 \%$ Extracellular region part

$\square .52 \%$ Proteinaceous extracellular matrix

$3.26 \%$ Postsynaptic density

16.30\% Unknown

Score $=-\operatorname{Lg}\left(P_{-}\right.$value $)$

$\mathrm{D}$

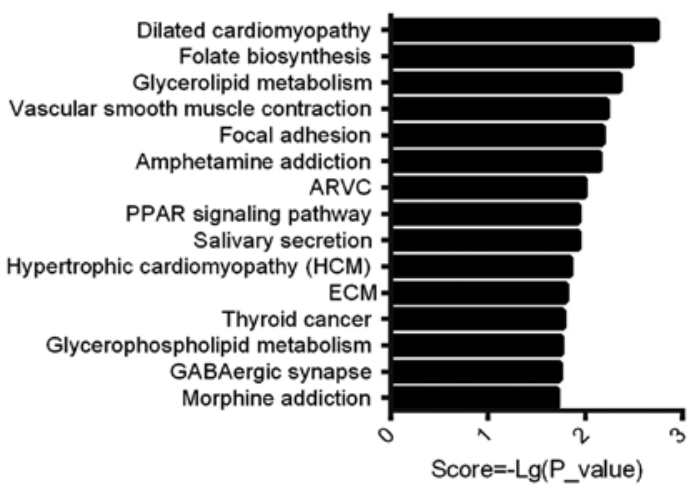

Figure 5. Bioinformatics analysis of $98 \mathrm{mRNAs}$ in DVT. GO analysis assigns high-level terms from each of the following three major GO terms: (A) Biological processes, (B) cellular components, and (C) molecular functions. (D) The top 15 canonical pathways [with score $>1.7$, P<0.02]. mRNA, messenger RNA; DVT, deep vein thrombosis; GO, gene ontology; ACA, adenylate cyclase activity; ARVC, arrhythmogenic right ventricular cardiomyopathy.
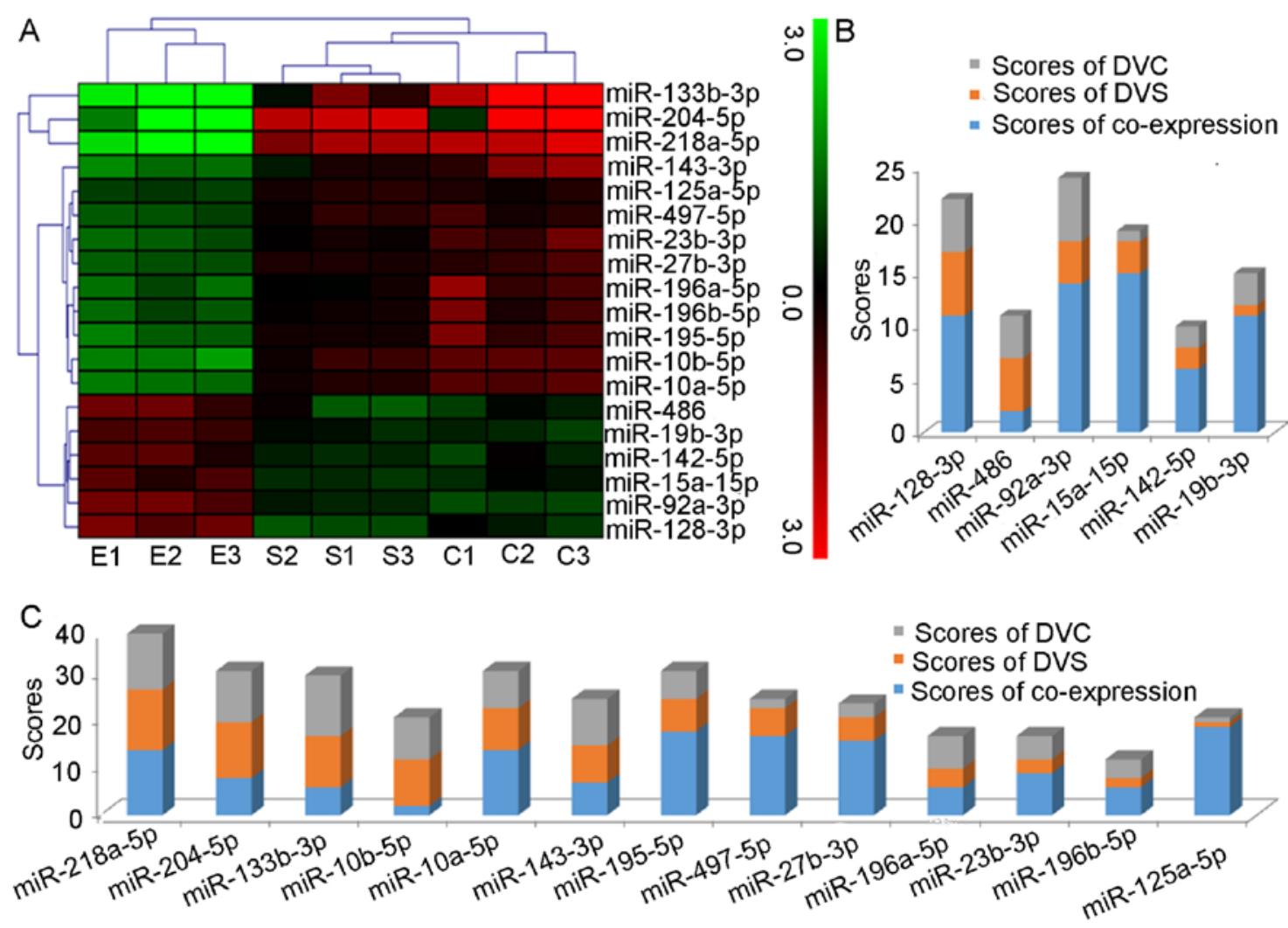

Figure 6. Bioinformatics analysis of 19 miRNAs in DVT. (A) Hierarchical cluster analysis of the 19 differentially expressed miRNAs. Hierarchical clustering analysis separated the nine samples into three groups, which were consistent with the DVT, control and sham groups. Red bars indicate high expression levels, green bars indicate low expression levels, and black bars indicate similar expression levels. (B) Scores of 6 upregulated and (C) 13 downregulated miRNAs. The scores are based on the degree of co-expression and the fold change. miRNA, micro RNA; DVT, deep vein thrombosis; FD-DVS, fold change in the DVT vs. sham groups; FD-DVC, fold change in the DVT vs. control groups. 
Table III. Differential expression of 6 upregulated miRNAs in the DVT, the control and the sham group.

\begin{tabular}{|c|c|c|c|c|c|}
\hline \multirow[b]{2}{*}{ miRNA } & \multicolumn{3}{|c|}{ Signal values (means $\pm \mathrm{SD}$ ) } & \multicolumn{2}{|c|}{ Fold change (P-value) } \\
\hline & DVT group & Control group & sham group & DVT/control & DVT/sham \\
\hline miR-19b-3p & $573.02 \pm 42.12$ & $229.30 \pm 24.47$ & $273.82 \pm 34.41$ & $2.50(1.14 \mathrm{E}-03)$ & $2.09(5.09 \mathrm{E}-03)$ \\
\hline miR-142-5p & $104.09 \pm 22.38$ & $45.03 \pm 11.02$ & $42.85 \pm 2.04$ & $2.31(2.52 \mathrm{E}-02)$ & $2.43(3.16 \mathrm{E}-02)$ \\
\hline $\operatorname{miR}-15 a-5 p$ & $185.14 \pm 32.59$ & $89.34 \pm 11.84$ & $72.66 \pm 56.24$ & $2.07(1.40 \mathrm{E}-02)$ & $2.55(1.44 \mathrm{E}-02)$ \\
\hline miR-92a-3p & $769.03 \pm 111.47$ & $190.20 \pm 9.65$ & $258.05 \pm 14.72$ & $4.04(3.17 \mathrm{E}-03)$ & $2.98(5.12 \mathrm{E}-03)$ \\
\hline $\mathrm{miR}-486$ & $826.37 \pm 182.19$ & $303.27 \pm 55.64$ & $263.43 \pm 112.57$ & $2.72(1.21 \mathrm{E}-02)$ & 3.14 (2.93E-02) \\
\hline $\operatorname{miR}-128-3 p$ & $396.74 \pm 52.95$ & $52.95 \pm 25.23$ & $88.78 \pm 4.53$ & 2.91 (3.89E-03) & $4.47(1.61 \mathrm{E}-03)$ \\
\hline
\end{tabular}

miRNA, micro RNA; DVT, deep vein thrombosis.

Table IV. Differential expression of 13 downregulated miRNAs in the DVT, the vontrol and the sham group.

\begin{tabular}{|c|c|c|c|c|c|}
\hline \multirow[b]{2}{*}{ miRNA } & \multicolumn{3}{|c|}{ Signal values (means $\pm \mathrm{SD}$ ) } & \multicolumn{2}{|c|}{ Fold change (P-value) } \\
\hline & DVT group & Control group & Sham group & DVT/Control & DVT/sham \\
\hline miR-218a-5p & $1.03 \pm 0.73$ & $58.88 \pm 9.69$ & $40.44 \pm 6.74$ & 56.94 (2.92E-02) & $39.11(3.41 \mathrm{E}-02)$ \\
\hline miR-204-5p & $0.73 \pm 0.87$ & $29.89 \pm 18.58$ & $28.45 \pm 2.91$ & 40.75 (3.01E-02) & 38.79 (3.93E-02) \\
\hline miR-133b-3p & $1.79 \pm 1.71$ & $1631.01 \pm 1898.14$ & $47.74 \pm 22.25$ & $912.16(1.00 \mathrm{E}-02)$ & $26.70(4.85 \mathrm{E}-02)$ \\
\hline miR-10b-5p & $75.88 \pm 9.55$ & $488.90 \pm 0.00$ & $333.18 \pm 53.56$ & 6.44 (2.48E-03) & 4.39 (9.00E-04) \\
\hline miR-10a-5p & $113.96 \pm 6.70$ & $569.35 \pm 34.06$ & $365.225 \pm 26.42$ & $5.00(1.11 \mathrm{E}-05)$ & $3.20(9.26 \mathrm{E}-05)$ \\
\hline miR-143-3p & $807.53 \pm 91.71$ & $5490.83 \pm 1852.49$ & $2341.94 \pm 438.29$ & $6.80(1.54 \mathrm{E}-02)$ & $2.90(6.51 \mathrm{E}-03)$ \\
\hline miR-195-5p & $169.80 \pm 23.48$ & $793.40 \pm 215.98$ & $456.61 \pm 0.00$ & $4.67(5.20 \mathrm{E}-03)$ & $2.69(1.06 \mathrm{E}-02)$ \\
\hline $\operatorname{miR}-497-5 p$ & $121.73 \pm 10.93$ & $331.66 \pm 56.00$ & $308.19 \pm 39.63$ & $2.72(4.81 \mathrm{E}-03)$ & $2.53(2.33 \mathrm{E}-03)$ \\
\hline $\operatorname{miR}-27 b-3 p$ & $245.33 \pm 14.03$ & $768.64 \pm 108.02$ & $605.37 \pm 8.52$ & $3.13(2.67 \mathrm{E}-03)$ & $2.47(1.26 \mathrm{E}-03)$ \\
\hline miR-196a-5p & $32.02 \pm 5.81$ & $157.60 \pm 58.56$ & $74.12 \pm 5.96$ & $4.92(1.21 \mathrm{E}-02)$ & $2.31(9.65 \mathrm{E}-03)$ \\
\hline miR-23b-3p & $421.90 \pm 49.45$ & $1712.503 \pm 358.92$ & $972.26 \pm 53.57$ & $4.06(2.97 \mathrm{E}-03)$ & $2.30(3.16 \mathrm{E}-03)$ \\
\hline miR-196b-5p & $31.71 \pm 4.44$ & $120.13 \pm 38.66$ & $70.53 \pm 3.39$ & $3.79(1.75 \mathrm{E}-02)$ & $2.22(8.43 \mathrm{E}-03)$ \\
\hline miR-125a-5p & $70.06 \pm 2.92$ & $142.10 \pm 10.40$ & $150.30 \pm 9.76$ & $2.03(1.14 \mathrm{E}-03)$ & $2.15(4.62 \mathrm{E}-04)$ \\
\hline
\end{tabular}

miRNA, micro RNA; DVT, deep vein thrombosis.

\section{Discussion}

DVT and PE are significant public health concerns, representing major sources of mortality and morbidity. Animal models are important for understanding the pathophysiology of these thrombogenesis-associated diseases (15). To minimize the error caused by surgery, a rat IVC ligation model was used in the present study, which provides a total stasis environment and a consistent thrombus size after 3 days of ligation. This model has been widely used in previous studies $(1,2,16-19)$.

Although miRNA screening methodologies have become widely available, and large studies of the role of miRNAs were performed in the pathogenesis of various cardiovascular diseases (20-24), to the best of our knowledge, only three studies reported miRNA profiling in vein thrombosis. Xiao et al (25) identified markedly higher plasma levels of various miRNAs, (including miR-134, miR-410 and miR-520 amongst others) in patients suffering from acute PE (25). The
miR-320a and miR-320b were upregulated in plasma samples from VTE patients compared with healthy controls (26). Qin et al (27) demonstrated an increased serum level of three miRNAs (miR-582, miR-195 and miR-532) in patients with postoperative DVT versus control subjects (27).

All previous studies identified differentially expressed miRNAs in the thrombosis group, consistent with the present study, but the specific miRNA profiles differed, which may be associated with differences in the subsets of selected diseases, species and types of samples that were evaluated. Beyond this, all of the known miRNA profiles associated with thrombosis were detected in plasma or serum as potential markers, while, to the best of our knowledge, there are no studies evaluating the miRNA profiles in venous tissue. The miRNA profiles from biological fluid are used primarily for diagnoses, while miRNA profiles in venous tissues may be more conducive to investigating the underlying mechanism of DVT, which has rarely been the focus of study. Therefore, the present study was designed to investigate this further. 


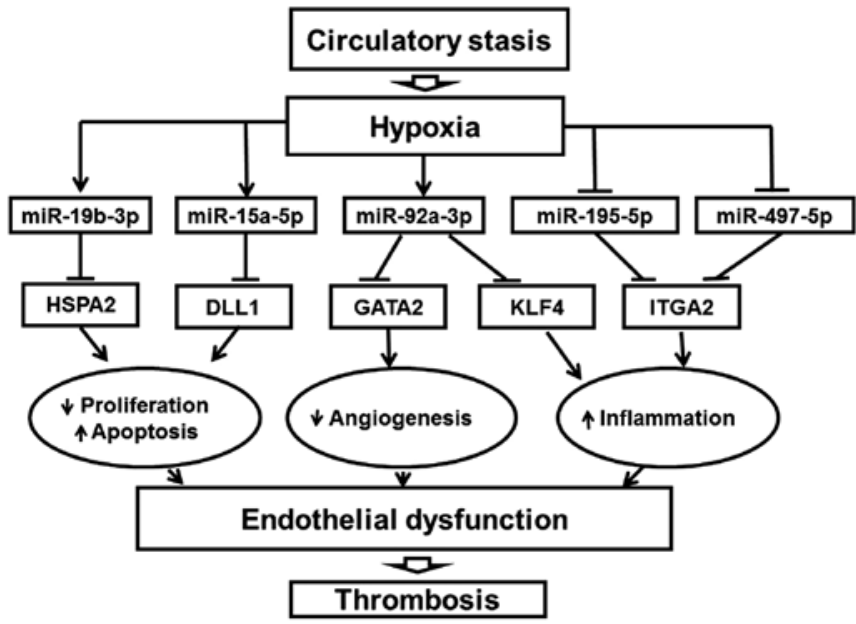

Figure 7. Role of endothelial miRNAs in DVT. The negative regulatory association between miRNAs and their predicted target genes was derived from the integrated analysis in the present study. The role and mechanisms of target genes in cell proliferation, apoptosis, angiogenesis and inflammation have been reported in part. Arrows indicate positive regulation and horizontal bars indicate negative regulation. miRNA, microRNA; DVT, deep vein thrombosis; HSPA2, heat shock protein family A (Hsp70) member 2; DLL1, delta like canonical Notch ligand 1; GATA2, GATA binding protein 2; KLF4, Kruppel like factor 4; ITGA2, integrin subunit $\alpha 2$.

By integrative methodology, miRNA/mRNA pairs in DVT were used to construct the regulatory network, indicating that these miRNAs may be important in DVT by regulating their target genes. Further GO and KEGG analyses of 98 genes indicated that DVT was associated with specific biological processes, such as angiogenesis or inflammation.

It is worth noting that the changes in these genes were reported to affect endothelial function $(28,29)$. For example, heat shock protein family A (Hsp70) member 2 (HSPA2) in the adult corneal endothelium were demonstrated to be sensitized to mediators of cell death (30). In addition, delta like canonical Notch ligand 1 (DLL1) is an essential Notch ligand in the vascular endothelium, and activates Notch1 to maintain arterial integrity (31). Another example is the angiogenic factor, GATA binding protein 2 (GATA2). Previous studies $(32,33)$ have indicated that GATA2 is important for vascular integrity. In addition, KLF4 functions as an important regulator of EC inflammation (34). Integrin subunit $\alpha 2$ (ITGA2), the major collagen-binding $\alpha$-integrin subunit in ECs, is involved in inflammatory processes and cell invasion $(35,36)$. Increased expression levels of ITGA2 increases joint inflammation (36).

Clustering analysis of 19 miRNAs revealed a distinct miRNA signature during DVT. Notably, the majority of the 19 miRNAs have also been reported in the endothelium. For example, miR-10b-5p and miR-195 have been identified as diagnostic markers of VTE $(26,27)$. In addition, miR-92a, miR-15a, miR-196a/196b and miR-19b have been reported to be involved in the processes of EC dysfunction, proliferation, apoptosis, migration and angiogenesis, which ultimately influence diseases, such as thrombosis and atherosclerosis, and tumors (37-41).

Maintenance of the functional integrity of the endothelium is important to preserve blood flow and prevent thrombosis (42). The endothelium secretes factors that control vascular relaxation and contraction, thermogenesis, fibrinolysis, and platelet activation and inhibition (43). While EC injury and dysfunction are considered to be the initial events in the development of thromboembolism, atherosclerosis, postangioplasty restenosis and plaque erosion contribute to macrovascular complications (44). The present findings indicate that endothelial dysfunction is particularly significant in ligation-induced thrombosis. A flowchart of the role of endothelial miRNAs in DVT was generated by integrating the expression and regulation of endothelial-associated miRNAs and target genes (Fig. 7).

In conclusion, profiling of miRNA and mRNA expression was performed in parallel to integrate the two biological levels to better understand DVT. The use of this integrated approached elucidates the associations between the expression levels of miRNAs and mRNAs. This novel systematic study provides information on miRNA target regulation and provides a foundation for future studies.

\section{Acknowledgements}

The authors would like to thank Binbin Zou for assistance with bioinformatics analysis. The present study was supported by grants from the National Natural Science Foundation of China (grant no. 81470088).

\section{References}

1. Tapson VF and Humbert M: Incidence and prevalence of chronic thromboembolic pulmonary hypertension: From acute to chronic pulmonary embolism. Proc Am Thorac Soc 3: 564-567, 2006.

2. Wang W, Li C, Li W, Kong L, Qian A, Hu N, Meng Q and Li X: MiR-150 enhances the motility of EPCs in vitro and promotes EPCs homing and thrombus resolving in vivo. Thromb Res 133: 590-598, 2014.

3. Heit JA: The epidemiology of venous thromboembolism in the community. Arterioscler Thromb Vasc Biol 28: 370-372, 2008.

4. Nosaka M, Ishida Y, Kimura A, Hama M, Kawaguchi T, Yamamoto H, Kuninaka Y, Shimada E and Kondo T: Immunohistochemical detection of intrathrombotic IL-6 and its application to thrombus age estimation. Int J Legal Med 129: 1021-1025, 2015

5. Tamaki $\mathrm{H}$ and Khasnis A: Venous thromboembolism in systemic autoimmune diseases: A narrative review with emphasis on primary systemic vasculitides. Vasc Med 20: 369-376, 2015.

6. Bartel DP: MicroRNAs: Genomics, biogenesis, mechanism, and function. Cell 116: 281-297, 2004.

7. Wang X, Sundquist K, Elf JL, Strandberg K, Svensson PJ, Hedelius A, Palmer K, Memon AA, Sundquist J and Zöller B: Diagnostic potential of plasma microRNA signatures in patients with deep-vein thrombosis. Thromb Haemost 116: 328-336, 2016.

8. Tan M, Yan HB, Li JN, Li WK, Fu YY, Chen W and Zhou Z: Thrombin stimulated platelet-derived exosomes inhibit plateletderived growth factor receptor-beta expression in vascular smooth muscle cells. Cell Physiol Biochem 38: 2348-2365, 2016.

9. Feinberg MW and Moore KJ: MicroRNA regulation of atherosclerosis. Circ Res 118: 703-720, 2016.

10. Chistiakov DA, Orekhov AN and Bobryshev YV: The role of miR-126 in embryonic angiogenesis, adult vascular homeostasis, and vascular repair and its alterations in atherosclerotic disease. J Mol Cell Cardiol 97: 47-55, 2016.

11. Fang Y and Davies PF: Site-specific microRNA-92a regulation of Kruppel-like factors 4 and 2 in atherosusceptible endothelium. Arterioscler Thromb Vasc Biol 32: 979-987, 2012.

12. Ebnet K, Aurrand-Lions M, Kuhn A, Kiefer F, Butz S, Zander K, Meyer zu Brickwedde MK, Suzuki A, Imhof BA and Vestweber D: The junctional adhesion molecule (JAM) family members JAM-2 and JAM-3 associate with the cell polarity protein PAR-3: A possible role for JAMs in endothelial cell polarity. J Cell Sci 116: 3879-3891, 2003.

13. Rafii S and Nolan D: Cholesterol activates vascular niche and hematopoiesis. Blood 115: 3857-3858, 2010. 
14. Regard JB, Scheek S, Borbiev T, Lanahan AA, Schneider A Demetriades AM, Hiemisch H, Barnes CA, Verin AD and Worley PF: Verge: A novel vascular early response gene. J Neurosci 24: 4092-4103, 2004.

15. Diaz JA, Obi AT, Myers DD Jr, Wrobleski SK, Henke PK, Mackman N and Wakefield TW: Critical review of mouse models of venous thrombosis. Arterioscler Thromb Vasc Biol 32: 556-562, 2012.

16. Diaz JA, Ballard-Lipka NE, Farris DM, Hawley AE, Wrobleski SK, Myers DD, Henke PK, Lawrence DA and Wakefield TW: Impaired fibrinolytic system in ApoE gene-deleted mice with hyperlipidemia augments deep vein thrombosis. J Vasc Surg 55: 815-822, 2012.

17. Nakata $\mathrm{N}$ and Kira Y: Effects of preoperative glycyrrhizin infusion for the prevention of venous thrombosis on the tissue expression of antithrombin in a rat model. Ann Vasc Dis 9: 95-101, 2016.

18. Oboshi M, Naito Y, Sawada H, Hirotani S, Iwasaku T, Okuhara Y, Morisawa D, Eguchi A, Nishimura K, Fujii K, et al: Temporary dietary iron restriction affects the process of thrombus resolution in a rat model of deep vein thrombosis. PLoS One 10: e0126611, 2015.

19. Yin M, Tian S, Huang X, Huang Y and Jiang M: Role and mechanism of tissue plasminogen activator in venous wall fibrosis remodeling after deep venous thrombosis via the glycogen synthase kinase-3 beta signaling pathway. J Surg Res 184: 1182-1195, 2013

20. Yepes S, López R, Andrade RE, Rodriguez-Urrego PA, López-Kleine L and Torres MM: Co-expressed miRNAs in gastric adenocarcinoma. Genomics 108: 93-101, 2016.

21. Setién-Olarra A, Bediaga NG, Acha-Sagredo A, MarichalarMendia X, de Pancorbo MM and Aguirre-Urizar JM Genomewide miRNA profiling of oral lichenoid disorders and oral squamous cell carcinoma. Oral Dis 22: 754-760, 2016.

22. Louwies T, Vuegen C, Panis LI, Cox B, Vrijens K, Nawrot TS and De Boever P: miRNA expression profiles and retinal blood vessel calibers are associated with short-term particulate matter air pollution exposure. Environ Res 147: 24-31, 2016.

23. Yentrapalli R, Azimzadeh O, Kraemer A, Malinowsky K, Sarioglu H, Becker KF, Atkinson MJ, Moertl S and Tapio S: Quantitative and integrated proteome and microRNA analysis of endothelial replicative senescence. J Proteomics 126: 12-23, 2015

24. Murphy MS, Casselman RC, Tayade C and Smith GN: Differential expression of plasma microRNA in preeclamptic patients at delivery and 1 year postpartum. Am J Obstet Gynecol 213: 367.e361-369, 2015.

25. Xiao J, Jing ZC, Ellinor PT, Liang D, Zhang H, Liu Y, Chen X, Pan L, Lyon R, Liu Y, et al: MicroRNA-134 as a potential plasma biomarker for the diagnosis of acute pulmonary embolism. J Transl Med 9: 159, 2011.

26. Starikova I, Jamaly S, Sorrentino A, Blondal T, Latysheva N, Sovershaev M and Hansen JB: Differential expression of plasma miRNAs in patients with unprovoked venous thromboembolism and healthy control individuals. Thromb Res 136: 566-572, 2015.

27. Qin J, Liang H, Shi D, Dai J, Xu Z, Chen D, Chen X and Jiang Q: A panel of microRNAs as a new biomarkers for the detection of deep vein thrombosis. J Thromb Thrombolysis 39: 215-221, 2015.

28. Johnson KE and Wilgus TA: Vascular endothelial growth factor and angiogenesis in the regulation of cutaneous wound repair. Adv Wound Care (New Rochelle) 3: 647-661, 2014.

29. Healy AM, Schwartz JJ, Zhu X, Herrick BE, Varnum B and Farber HW: Gas 6 promotes Axl-mediated survival in pulmonary endothelial cells. Am J Physiol Lung Cell Mol Physiol 280: L1273-L1281, 2001.
30. Frausto RF, Wang $\mathrm{C}$ and Aldave AJ: Transcriptome analysis of the human corneal endothelium. Invest Ophthalmol Vis Sci 55: 7821-7830, 2014

31. Sörensen I, Adams RH and Gossler A: DLL1-mediated Notch activation regulates endothelial identity in mouse fetal arteries. Blood 113: 5680-5688, 2009.

32. Johnson KD, Hsu AP, Ryu MJ, Wang J, Gao X, Boyer ME, Liu Y, Lee Y, Calvo KR, Keles S, et al: Cis-element mutated in GATA2-dependent immunodeficiency governs hematopoiesis and vascular integrity. J Clin Invest 122: 3692-3704, 2012.

33. Lim KC, Hosoya T, Brandt W, Ku CJ, Hosoya-Ohmura S, Camper SA, Yamamoto $M$ and Engel JD: Conditional Gata2 inactivation results in HSC loss and lymphatic mispatterning. J Clin Invest 122: 3705-3717, 2012.

34. Hamik A, Lin Z, Kumar A, Balcells M, Sinha S, Katz J, Feinberg MW, Gerzsten RE, Edelman ER and Jain MK: Kruppel-like factor 4 regulates endothelial inflammation. J Biol Chem 282: 13769-13779, 2007.

35. Roche F, Sipilä K, Honjo S, Johansson S, Tugues S, Heino J, Claesson-Welsh L: Histidine-rich glycoprotein blocks collagenbinding integrins and adhesion of endothelial cells through low-affinity interaction with $\alpha 2$ integrin. Matrix Biol 48: 89-99, 2015.

36. Peters MA, Wendholt D, Strietholt S, Frank S, Pundt N, Korb-Pap A, Joosten LA, van den Berg WB, Kollias G, Eckes B, et al: The loss of $\alpha 2 \beta 1$ integrin suppresses joint inflammation and cartilage destruction in mouse models of rheumatoid arthritis. Arthritis Rheum 64: 1359-1368, 2012.

37. Sandrim VC, Dias MC, Bovolato AL, Tanus-Santos JE, Deffune E and Cavalli RC: Plasma from pre-eclamptic patients induces the expression of the anti-angiogenic miR-195-5p in endothelial cells. J Cell Mol Med 20: 1198-1200, 2016.

38. Yu S, Hong Q, Wang Y, Hou K, Wang L, Zhang Y, Fu B, Zhou Y, Zheng W, Chen X, et al: High concentrations of uric acid inhibit angiogenesis via regulation of the Kruppel-like factor 2-vascular endothelial growth factor-A axis by miR-92a. Circ J 79: 2487-2498, 2015.

39. Xue Y, Wei Z, Ding H, Wang Q, Zhou Z, Zheng S, Zhang Y, Hou D, Liu Y, Zen K, et al: MicroRNA-19b/221/222 induces endothelial cell dysfunction via suppression of PGC-1 $\alpha$ in the progression of atherosclerosis. Atherosclerosis 241: 671-681, 2015.

40. Zheng X, Li A, Zhao L, Zhou T, Shen Q, Cui Q and Qin X: Key role of microRNA-15a in the KLF4 suppressions of proliferation and angiogenesis in endothelial and vascular smooth muscle cells. Biochem Biophys Res Commun 437: 625-631, 2013.

41. Plummer PN, Freeman R, Taft RJ, Vider J, Sax M, Umer BA, Gao D, Johns C, Mattick JS, Wilton SD, et al: MicroRNAs regulate tumor angiogenesis modulated by endothelial progenitor cells. Cancer Res 73: 341-352, 2013.

42. Lüscher TF and Barton M: Biology of the endothelium. Clin Cardiol 20 (Suppl 2): II-3-II-10, 1997.

43. Axtell AL, Gomari FA and Cooke JP: Assessing endothelial vasodilator function with the Endo-PAT 2000. J Vis Exp: Oct 15 , 2010 (Epub ahead of print). doi: 10.3791/2167.

44. Hartge MM, Kintscher U and Unger T: Endothelial dysfunction and its role in diabetic vascular disease. Endocrinol Metab Clin North Am 35: 551-560, viii-ix, 2006. 